\title{
Application of Constrained Optimization Approach to Missing Data in Experimental Design
}

\author{
Michael Ekholuenetale ${ }^{1}$, Adamson O. Ajakaiye ${ }^{2}$ \\ ${ }^{1}$ Women's Health and Action Research Centre, Km11, Benin-Lagos Expressway, Igue-Iheya, Benin City. Nigeria \\ ${ }^{2}$ Department of Mathematics, Faculty of Physical Sciences, University of Benin, Nigeria
}

\begin{abstract}
During the course of a research data obtained may be fully observed or particularly observed. If the data obtained from a research is partially observed, then a common problem in experiment has occurred. This problem is known as the "missing observation". Missing observation infers that no data (value) is stored for the variable in the current observation. Missing data are recurring in all sorts of research irrespective of the field; science, medical, agricultural and social science and so on. Researchers are faced with the problem of partially observed data sets. There are several reasons why data may be missing. They may be missing due to failure to record, gross errors in recording, accident and death amongst others. Missing data are very sensitive issues and many analyses techniques cannot proceed with gaps in their data. These missing values must be estimated and replaced before the analysis can be completed.
\end{abstract}

Keywords: Constrained Optimization, Missing observation, ANOVA, Design of Experiment

\section{Introduction}

Data set with gaps in their values are referred to as partially observed data sets, while those without gaps are known as fully observed data [1]. There are many types of missing data and different reasons for the data being missing and both issues affect the analysis. Some examples are enumerated below:

1) In a postal questionnaire survey not all the selected individuals respond.

2) Some laboratory samples are lost in transit or technically unsatisfactory.

3) Some experimental units may be lost as a result of death or other causes.

4) In a randomized trial some patients are lost during follow up stages before the end of the study.

5) In a multi-centre study some centres do not measure a particular variable.

6) Occasionally, data are missing because some equipment failed.

7) In a study assessing quality of life some patients die during the follow up period [2][3].

The prime concern is always, whether the available data would be biased. The fact that an observation is missing is unrelated to both the unobserved value and the data that are available. This is called "missing completely at random [4]. Sometimes data are missing in a predictable way that does not depend on the missing values itself but which can be predicted from other data and this is called "missing at random"'[5].

It is worthy of note that the missing data probably depend on the unobserved values and this is known as "missing not at random" [4][5][6]. Therefore, the absence of these data may lead to biased results. Furthermore there are other types of missing data:

1) Missing by definition of the subpopulation

2) Non ignorable missing values

3) Deliberate removal of outlier i.e. outrageous value [7]
Missing observations are handled in different ways. There are different approaches and software for working with missing observations. These approaches can be traditional or modern/alternative approach.

The traditional approach includes method like;

1) List wise deletion

2) Pair wise deletion

3) Mean substitution

While the modern/alternative approach includes methods like:

1) Constrained optimization

2) Single imputation

3) Multiple imputation

Srivastava [8] postulated that any time the number of observation is not the same for all factors, level combination, such an experimental design is unbalanced thus an experiment or design with one or more missing observation is an unbalanced design or experiment.

Sequel to the above, there are various reasons attributable to missing data/observations they include amongst others:

1) Faulty equipment

2) Error of transposition (data not correctly entered)

3) Due to carelessness (where record are not well kept hence data misses)

4) Uncontrollable weather condition

5) Relocation of person (s) with the access to a said data (traveling out)

6) Death before recording

7) Power failure on timing experiment

This study is aimed to achieve the following:

1) To strengthen our understanding in the theoretical aspect of working with partially observed data sets in a randomized complete block experiment.

2) To bring the applications of formula for missing values to play on real life occurrence of missing data 


\section{International Journal of Science and Research (IJSR) \\ ISSN (Online): 2319-7064}

Index Copernicus Value (2013): 6.14 | Impact Factor (2015): 6.391

\section{Constrained Optimization Approach}

When working with missing observations in an experimental design, there are several methods to apply. The constrained optimization is one of the most commonly used methods. It was developed by Yates Frank in 1933. It estimates missing values in experimental design by making use of the parameter in the mathematical model of the design. In a randomized complete block experiment, the constrained optimization approach makes use of treatment and the block (Replication) effect parameter in the mathematical model. The constrained optimization approach gives a good estimate of missing values. If the number of block (Replications) and treatment are considerably large when more than one value is missing the constrained optimization employs a rather crude cyclic method [1][2][10].

\section{Method}

\section{Optimization of the Mathematical Model}

The most common method for estimating missing observations is the Yates formulas for missing values. Yates obtained this formula by optimizing the linear model for a design using the constrained optimization approach. The constrained optimization approach optimizes a function subject to some certain constraints [10].

\section{Procedural Steps for Constrained Optimization}

Given a function $\mathrm{f}(\mathrm{x})$ : It is optimized by the following procedure;

First; form a function $\phi(x)$ say

$$
\phi(x)=f(x)+\lambda_{1} g_{1}(x)+\lambda_{2} g_{2}(x)
$$

Then optimize $\phi(x)$ with respect to $\chi, \lambda_{1}$ and $\lambda_{2}$. $\lambda_{1}$ and $\lambda_{2}$ are called langrage (undetermined) multipliers that is put $\frac{\partial \phi(x)}{\partial x}=0, \frac{\partial \phi(x)}{\partial \lambda_{1}}=0$, and $\frac{\partial \phi(x)}{\partial \lambda_{2}}=0$

\section{Model of Randomized Complete Block Design}

Given that observation $X_{11}$ is missing, the linear model is optimized and an estimate for $\mathrm{X}_{11}$ is obtained thus:

$$
\begin{aligned}
& X_{i j}=\mu+b_{j}+t_{i}+\varepsilon_{i j} \\
& X_{i j}-\mu-b_{j}-t_{i}=\varepsilon_{i j} \\
& \varepsilon_{i j}=X_{i j}-\mu-b_{j}-t_{i}
\end{aligned}
$$

Now,

Let

$$
Z=\sum_{i}^{t} \sum_{j}^{b}\left(X_{i j}-\mu-b_{j}-t_{i}\right)^{2}=\sum_{i} \sum_{j} \varepsilon_{i j}^{2}
$$

Subject to

$$
\sum_{j} b_{j}=\sum_{i}^{t} t_{i}=0
$$

Where we form $\phi(x)$
Since $X_{11}$ is missing, which means treatment in block 1 is missing

$$
\begin{aligned}
& \mathrm{i} \neq 1, \mathrm{j} \neq 1 \\
& \partial \phi(x)=-2 \sum_{i \neq 1} \sum_{j \neq 1}\left(X_{i j}-\mu-b_{j}-t_{i}\right)=0 \\
& =\sum_{i \neq 1} \sum_{j \neq 1}\left(X_{i j}-\mu-b_{j}-t_{i}\right)=0 \\
& =\sum_{i \neq 1} \sum_{j \neq 1} X_{i j}-\sum_{i \neq 1}^{t} \sum_{j \neq 1}^{b} \mu-\sum_{i \neq 1}^{t} \sum_{j \neq 1}^{b} b_{j}-\sum_{i=1}^{t} \sum_{j \neq 1}^{b} t_{i}=0 \\
& =\sum_{i=1}^{t} \sum_{j \neq 1}^{b} X_{i j}-\left(b_{k}\right) \mu-\sum_{i \neq 1}^{t} \sum_{j \neq 1}^{b} b_{j}-\sum_{i=1}^{t} \sum_{j \neq 1}^{b} t_{i}=0 \\
& \frac{\partial \phi(x)}{\partial \mu}=T-\left(b_{k}\right) \mu+b_{i}+t_{i}=0 \text { (1) } \\
& \frac{\partial \phi(x)}{\partial \mu}=T-\left(b_{k-1}\right) \mu+b_{i}+t_{i}=0
\end{aligned}
$$

Now;

$$
\begin{gathered}
b_{1}=\sum_{i+1} \sum_{j+1} b_{j} \sum_{i+1} \sum_{j+1} b_{j} \\
=0-\sum_{i+1} \sum_{j+1} b_{j} \\
\therefore b_{1}=-\sum_{i+1} \sum_{j+1} b_{j}
\end{gathered}
$$

Also,

$$
\begin{aligned}
& t_{1}=\sum_{i \neq 1} \sum_{j \neq 1} t_{i} \sum_{i \neq 1} \sum_{j \neq 1} t_{i} \\
& \quad=0-\sum_{i \neq 1} \sum_{j \neq 1} t_{i} \\
& \therefore t_{i}=-\sum_{i \neq 1} \sum_{j \neq 1} t_{i} \\
& \phi(x)=\sum_{i \neq 1} \sum_{j \neq 1}\left(X_{i j}-\mu-b_{j}-t_{i}\right)^{2}+2 \lambda \sum_{j} b_{j}+2 \lambda_{2} \sum_{i} t_{i}
\end{aligned}
$$

Where,

$$
\begin{aligned}
& j=1 \phi(s) i s \\
& \phi(x)=\sum_{i \neq 1}\left(X_{i j}-m-b_{1}-t_{i}\right)^{2}+2 x_{i} b_{i}+2 \lambda_{2} \sum_{i \neq 1} t_{i} \\
& \frac{\partial \phi(x)}{\partial b_{1}}=-2 \sum_{i \neq 1}\left(X_{i j}-\mu-b_{i}-t_{i}\right)+2 \lambda_{1}=0 \\
& =\sum_{i \neq 1}\left(X_{i j}-\mu-b_{i}-t_{i}\right)-\lambda_{1}=0 \\
& =\sum_{i \neq 1} X_{i 1}-\sum_{i \neq 1} \mu-\sum_{i \neq 1} b_{i}-\sum_{i \neq 1} t_{i}-\lambda_{1}=0
\end{aligned}
$$

i.e

$$
\frac{\partial \phi}{\partial b_{i}}=\beta_{i}-(t-1) \mu-(t-1) b_{i}+t_{i}-\lambda_{1}=0
$$

$$
\phi(x)=\sum_{i \neq 1} \sum_{j \neq 1}\left(X_{i j}-\mu-b_{j}-t_{i}\right)^{2}+2 \lambda_{1} \sum_{j \neq 1} b_{j}+2 \lambda_{2} \sum_{i \neq 1} t_{i}
$$

Note, however;

\section{Volume 5 Issue 9, September 2016}




$$
\begin{aligned}
& \sum_{i \neq 1} t_{i}=t_{j}+\sum_{i \neq 1} t_{i} \\
& \sum_{i \neq 1} t_{i}=\sum_{i \neq 1} t_{i}-t_{i} \\
& \sum_{i \neq 1} t_{i}=0-t_{i} \\
& \sum_{i \neq 1} t_{i}=t_{i}
\end{aligned}
$$

In the same way;

When $\mathrm{i}=1, \Phi(\mathrm{x})$ becomes:

$$
\phi(x)=\sum\left(X_{i j}-\mu-b_{j}-t_{i}\right)^{2}+2 \lambda_{1} \sum_{i \neq 1} b_{j}+2 \lambda_{2} t_{i}
$$

Also,

$$
\begin{aligned}
\frac{\partial \phi}{\partial t_{i}}=-2 & \sum_{j \neq 1}\left(X_{i j}-\mu-b_{j}-t_{i}\right)-\lambda_{2}=0 \\
= & \sum_{j \neq 1}\left(X_{i j}-\mu-b_{j}-t_{i}\right)-\lambda_{2}=0 \\
& =\sum_{j \neq 1} X_{i j}-\sum_{j \neq 1} \mu-\sum_{j \neq 1} b_{j}-\sum_{j \neq 1} t_{i}-\lambda_{2}=0 \\
\frac{\partial \phi}{\partial t_{i}} & =T_{i .}-(b-1) \mu .(b-1) t_{i}-b_{i}-\lambda_{2}=0
\end{aligned}
$$

When $\mathrm{j} \neq 1$, $\Phi(\mathrm{x})$ becomes;

$$
\begin{aligned}
\frac{\partial \phi(x)}{\partial b_{j \neq 1}}= & -2 \sum_{i}\left(X_{i j}-\mu-b_{j}-t_{i}\right)+2 \lambda_{1}=0 \\
& =\sum_{i}\left(X_{i j}-\mu-b_{j}-t_{i}\right)+2 \lambda_{1}=0 \\
& =\sum_{i} X_{i j}-\sum_{i} \mu-\sum_{i} b_{j}-\sum_{i} t_{i}-\lambda_{1}=0 \\
& \frac{\partial \phi(x)}{\partial b_{j \neq 1}}=\beta_{. j}-t \mu-t b_{j}-\lambda_{1}=0
\end{aligned}
$$

Also

When $\mathrm{i} \neq 1, \Phi(\mathrm{x})$ yield (becomes)

$$
\begin{gathered}
\phi(x)=\sum_{j}\left(X_{i j}-\mu-b_{j}-t_{i}\right)^{2}+2 \lambda \sum_{j \neq 1} b_{j}+2 \lambda_{2} \sum_{i \neq 1} t_{i} \\
\frac{\partial \phi(x)}{\partial b_{j \neq 1}}=-2 \sum_{j}\left(X_{i j}-\mu-b_{j}-t_{i}\right)+2 \lambda_{1}=0 \\
=\sum_{j}\left(X_{i j}-\mu-b_{j}-t_{i}\right)-\lambda_{2}=0 \\
=\sum_{j} X_{i j} \sum_{j} \mu-\sum_{j} b_{j}-\sum_{j} t_{i}-\lambda_{2}=0 \\
\frac{\partial \phi(x)}{\partial t_{j \neq 1}}=T_{i}-b \mu-b t_{i}-\lambda_{2}=0
\end{gathered}
$$

Solving equation (1) and (2) through equation (5) simultaneously, we have

$$
\lambda_{1}=0, \lambda_{2}=0 \Rightarrow \lambda_{1}=\lambda_{2}=0
$$

$$
\begin{aligned}
& \mu-\hat{b}_{1}+\hat{t}_{1}=\frac{b \hat{\beta}_{. j}+t \hat{T}_{i .}-T}{(b-1)(t-1)}=\hat{X}_{11} \\
& \therefore \hat{X}_{11}=\frac{b \hat{\beta}_{. j}+t \hat{T}_{i .}-T}{(b-1)(t-1)}
\end{aligned}
$$

When a unit is missing from a dataset, it is simply estimated using the Yates formula (one star formula). Thus, the general formula;

$$
\hat{X}_{i j}=\frac{b \hat{\beta} \cdot . j+t \hat{T}_{i .}-T}{(b-1)(t-1)}
$$

Where $\beta=$ total of the remaining unit in Block (replicates where the missing unit appears)

$\hat{T}=$ total yield of the particular treatment in the other blocks (replicates)

$\mathrm{T} . .=$ grand total

$\mathrm{t}=$ number of treatments

$\mathrm{b}=$ number of block (replicates)

However, we mention here that its only when a unit is missing, we make use of the one star general equation.

$$
\bar{X}_{R C}=\frac{b B_{\cdot_{C}}+t T_{r} \cdot-T}{(b-1)(t-1)}
$$

Assuming $\mathrm{R}=\mathrm{Row}=2^{\text {nd }}$ row and $\mathrm{C}=$ column $=3^{\text {rd }}$ column .

$$
\bar{X}_{23}=\frac{b B_{\cdot_{3}}+t T_{2} \cdot-T}{(b-1)(t-1)}
$$

When more than a value is missing in a data set, we use the crude cyclic method. The crude cyclic method estimates one value at a time after substituting arbitrary values for the other missing observations (i.e. taking a simple average from the rows until there is no difference between columns).

Assume $a, b, c, \ldots$ are missing we first givers values by inspection, for all units $(b, c, \ldots)$ except "a" the one star general formula is then used to find an estimate for $(\hat{a})$ i.e.

$$
\hat{X}=\frac{b B \cdot_{c}+t T_{r} \cdot-T}{(b-1)(t-1)}
$$

With this estimate for $\left(\begin{array}{l}a \\ a\end{array}\right)$ and the value previously assumed for $(\mathrm{c}, \ldots)$ we again use one star general formula above to find an estimate for $b$, with the estimate found for $a$ and $b$ we use one star general formula to estimate for $\left(\begin{array}{l}\wedge \\ c\end{array}\right)$

Hence

\section{Volume 5 Issue 9, September 2016 www.ijsr.net}




\section{International Journal of Science and Research (IJSR) \\ ISSN (Online): 2319-7064}

Index Copernicus Value (2013): 6.14 | Impact Factor (2015): 6.391

After a complete cycle of operations a second estimate $(\hat{a})$ is found for a and so on, until the new estimates

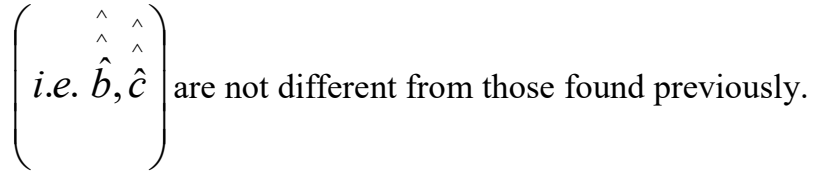

$$
(\text { i.e. } \hat{a}=\hat{a}, \hat{b}=\hat{b}, \hat{c}=\hat{c}, \ldots)
$$

However, there are cases which neither Yates formula nor the crude cyclic method can handle the cases are:

1. When a complete replicate of a treatment is missing

2. When a complete block (replicate) is missing

\section{Results}

Data obtained to determine the effect of varieties of feed on water consumption in a poultry farm.

Table 1: Effect of Feeds on Water Consumption

\begin{tabular}{|c|c|c|c|c|}
\hline Weeks & $\begin{array}{c}\text { Maize } \\
\text { Flour }\end{array}$ & $\begin{array}{c}\text { Maize Bran } \\
\text { Flour }\end{array}$ & $\begin{array}{c}\text { Beans } \\
\text { Flour }\end{array}$ & $\begin{array}{c}\text { Cassava } \\
\text { Flour }\end{array}$ \\
\hline Week 1 & $\mathrm{X}_{11}$ & 8.00 & 7.73 & 7.71 \\
\hline Week 2 & 9.60 & 8.15 & 7.87 & 7.24 \\
\hline Week 3 & 8.14 & 7.75 & $\mathrm{X}_{33}$ & 7.64 \\
\hline Week 4 & 7.76 & 7.42 & 7.80 & 8.24 \\
\hline Week 5 & 7.17 & 7.68 & 7.21 & 8.03 \\
\hline Week 6 & 7.46 & 8.20 & 7.67 & $\mathrm{X}_{64}$ \\
\hline
\end{tabular}

For: $X_{11}=9.2$

$\mathrm{X}_{33}=7.78$

$\mathrm{X}_{64}=8.23$

\section{Computation}

Using Yates formula

$$
\hat{X}_{i j}=\frac{b B_{. j}+t T_{t} \cdot-T}{(b-1)(t-1)}
$$

Where

$\mathrm{b}$ - denote the number of blocks (replicated)

$\hat{B}_{. j}$ - denote the total of the $j_{t h}$ block replicated

$\mathrm{t}$ - denote the number of treatment

$\mathrm{T}_{\mathrm{i}}$ - denote the total of the $i_{\text {th }}$ treatments

$\mathrm{T}$. . - is the grand mean

In an attempt to use the Yates formula to estimate a value for $\mathrm{X}_{11}$ a trial value was obtained for $\mathrm{X}_{33}$ and $\mathrm{X}_{64}$ respectively by taking a mean using the other members of theirs rows respective in both cases.

$$
\begin{aligned}
X_{33}= & \frac{7.14+7.75+7.64}{3}=\frac{23.53}{3} \\
& =7.843 \cong 7.84
\end{aligned}
$$

Also

$$
\begin{aligned}
X_{64} & =\frac{7.46+8.20+7.67}{3}=\frac{23.33}{3} \\
& =7.776 \cong 7.78
\end{aligned}
$$

To estimate $\hat{X}_{11}$ using Yates formula with the insertion of trial values for $\mathrm{X}_{33}$ and $\mathrm{X}_{66}$ to be 7.84 and 7.78 respectively.

Table 2: First missing observation

\begin{tabular}{|l|c|c|c|c|c|}
\hline Weeks & $\begin{array}{c}\text { Maize } \\
\text { Flour }\end{array}$ & $\begin{array}{c}\text { Maize Bran } \\
\text { Flour }\end{array}$ & $\begin{array}{c}\text { Beans } \\
\text { Flour }\end{array}$ & $\begin{array}{c}\text { Cassava } \\
\text { Flour }\end{array}$ & $B_{. j}$ \\
\hline Week 1 & $\mathrm{X}_{11}$ & 8.00 & 7.73 & 7.71 & 23.44 \\
\hline Week 2 & 9.60 & 8.15 & 7.87 & 7.24 & 32.86 \\
\hline Week 3 & 8.14 & 7.75 & 7.84 & 7.64 & 31.37 \\
\hline Week 4 & 7.76 & 7.42 & 7.80 & 8.24 & 31.22 \\
\hline Week 5 & 7.17 & 7.68 & 7.21 & 8.03 & 30.09 \\
\hline Week 6 & 7.46 & 8.20 & 7.67 & 7.78 & 31.11 \\
\hline $\mathrm{T}_{\mathrm{i} .}$ & $\mathbf{4 0 . 1 3}$ & $\mathbf{4 7 . 2 0}$ & $\mathbf{4 6 . 1 2}$ & $\mathbf{4 6 . 6 4}$ & $\mathbf{1 8 0 . 0 9}$ \\
\hline
\end{tabular}

Where number of treatment $=4$ (i.e. variety of feeds)

Where number of blocks (replicates) $=6$ (i.e. different weeks)

\begin{tabular}{|c|c|c|c|c|c|}
\hline Weeks & $\begin{array}{l}\text { Maize } \\
\text { Flour }\end{array}$ & $\begin{array}{l}\text { Maize Bran } \\
\text { Flour }\end{array}$ & $\begin{array}{l}\text { Beans } \\
\text { Flour }\end{array}$ & $\begin{array}{c}\text { Cassava } \\
\text { Flour }\end{array}$ & $B_{. j}$ \\
\hline Week 1 & 8.07 & 8.00 & 7.73 & 7.71 & 31.51 \\
\hline Week 2 & 9.60 & 8.15 & 7.87 & 7.24 & 32.86 \\
\hline Week 3 & 8.14 & 7.75 & $\hat{X}_{33}$ & 7.64 & 23.53 \\
\hline Week 4 & 7.76 & 7.42 & 7.80 & 8.24 & 31.22 \\
\hline Week 5 & 7.17 & 7.68 & 7.21 & 8.03 & 30.09 \\
\hline Week 6 & 7.46 & 8.20 & 7.67 & 7.78 & 31.11 \\
\hline $\mathrm{T}_{\mathrm{i} .}$ & 48.20 & 47.20 & 38.28 & 46.64 & 180.32 \\
\hline
\end{tabular}

Subtracting values ratio Yates formula

$$
\begin{gathered}
=\hat{X}_{11}=\frac{6(23.44)+4(40.13)-180.09}{(6-1)(4-1)} \\
=\frac{121.07}{15} \\
\hat{X}_{11}=8.071 \cong 8.07
\end{gathered}
$$

Inserting $\hat{X}_{11}=8.07$ into the above table 2 gave the dataset table 3 below:

Table 3: Output from first computation$$
=\hat{X}_{23}=\frac{6(23.53)+4(38.28)-180.32}{(6-1)(4-1)}
$$

$$
=\frac{113.98}{15}
$$

$\hat{X}_{11}=7.598 \cong 7.60$

Inserting $\hat{X}_{33}=7.60$ into table 3 above gave table 4 below:

Table 4: Output from second computation

\begin{tabular}{|c|c|c|c|c|c|}
\hline Weeks & $\begin{array}{c}\text { Maize } \\
\text { Flour }\end{array}$ & $\begin{array}{c}\text { Maize Bran } \\
\text { Flour }\end{array}$ & $\begin{array}{c}\text { Beans } \\
\text { Flour }\end{array}$ & $\begin{array}{c}\text { Cassava } \\
\text { Flour }\end{array}$ & $B_{. j}$ \\
\hline Week 1 & 8.07 & 8.00 & 7.73 & 7.71 & 31.51 \\
\hline Week 2 & 9.60 & 8.15 & 7.87 & 7.24 & 32.86 \\
\hline Week 3 & 8.14 & 7.75 & 7.60 & 7.64 & 31.13 \\
\hline Week 4 & 7.76 & 7.42 & 7.80 & 8.24 & 31.22 \\
\hline Week 5 & 7.17 & 7.68 & 7.21 & 8.03 & 30.09 \\
\hline Week 6 & 7.46 & 8.20 & 7.67 & $\hat{X}_{64}$ & 23.33 \\
\hline $\mathrm{T}_{\mathrm{i} .}$ & $\mathbf{4 8 . 2 0}$ & $\mathbf{4 7 . 2 0}$ & $\mathbf{4 5 . 8 8}$ & $\mathbf{3 8 . 8 6}$ & $\mathbf{1 8 0 . 1 4}$ \\
\hline
\end{tabular}




\section{International Journal of Science and Research (IJSR) \\ ISSN (Online): 2319-7064}

Index Copernicus Value (2013): 6.14 | Impact Factor (2015): 6.391

$$
\begin{gathered}
=\hat{X}_{64}=\frac{6(23.33)+4(38.86)-180.14}{(6-1)(4-1)} \\
=\frac{115.28}{15} \\
\hat{X}_{11}=7.685 \cong 7.69
\end{gathered}
$$

Inserting $\hat{X}_{64}=7.69$ into table 4 above gave table 5 below:

Table 5: Output from third computation

\begin{tabular}{|c|c|c|c|c|c|}
\hline Weeks & $\begin{array}{c}\text { Maize } \\
\text { Flour }\end{array}$ & $\begin{array}{c}\text { Maize Bran } \\
\text { Flour }\end{array}$ & $\begin{array}{c}\text { Beans } \\
\text { Flour }\end{array}$ & $\begin{array}{c}\text { Cassava } \\
\text { Flour }\end{array}$ & $B_{. j}$ \\
\hline Week 1 & $\hat{X}_{11}$ & 8.00 & 7.73 & 7.71 & 23.44 \\
\hline Week 2 & 9.60 & 8.15 & 7.87 & 7.24 & 32.86 \\
\hline Week 3 & 8.14 & 7.75 & 7.60 & 7.64 & 31.13 \\
\hline Week 4 & 7.76 & 7.42 & 7.80 & 8.24 & 31.22 \\
\hline Week 5 & 7.17 & 7.68 & 7.21 & 8.03 & 30.09 \\
\hline Week 6 & 7.46 & 8.20 & 7.67 & 7.69 & 31.02 \\
\hline $\mathrm{T}_{\mathrm{i} .}$ & $\mathbf{4 0 . 1 3}$ & $\mathbf{4 7 . 2 0}$ & $\mathbf{4 5 . 8 8}$ & $\mathbf{4 6 . 5 5}$ & $\mathbf{1 7 9 . 7 6}$ \\
\hline
\end{tabular}

$$
=\hat{X}_{11}=\frac{6(23.44)+4(40.13)-179.76}{(6-1)(4-1)}
$$

$$
=\frac{121.4}{15}
$$

\begin{tabular}{|c|c|c|c|c|c|}
\hline Weeks & $\begin{array}{l}\text { Maize } \\
\text { Flour }\end{array}$ & $\begin{array}{c}\text { Maize Bran } \\
\text { Flour }\end{array}$ & $\begin{array}{l}\text { Beans } \\
\text { Flour }\end{array}$ & $\begin{array}{c}\text { Cassava } \\
\text { Flour }\end{array}$ & $B_{. j}$ \\
\hline Week 1 & 8.09 & 8.00 & 7.73 & 7.71 & 31.53 \\
\hline Week 2 & 9.60 & 8.15 & 7.87 & 7.24 & 32.86 \\
\hline Week 3 & 8.14 & 7.75 & $\hat{\hat{X}}_{33}$ & 7.64 & 23.53 \\
\hline Week 4 & 7.76 & 7.42 & 7.80 & 8.24 & 31.22 \\
\hline Week 5 & 7.17 & 7.68 & 7.21 & 8.03 & 30.09 \\
\hline Week 6 & 7.46 & 8.20 & 7.67 & 7.69 & 31.02 \\
\hline $\mathrm{T}_{\mathrm{i} .}$ & 48.22 & 47.20 & 38.28 & 46.55 & 180.25 \\
\hline
\end{tabular}

$\hat{X}_{11}=8.09$

Inserting $\hat{X}_{11}=8.09$ into table 5 above gave the table 6 below:

Table 6: Output from fourth computation

$$
\hat{X}_{33}=\frac{6(23.53)+4(38.28)-180.25}{(6-1)(4-1)}
$$

$$
=\frac{114.05}{15}
$$

$$
\hat{X}_{33}=7.603 \cong 7.60
$$

Inserting $\hat{X}_{33}=7.60$ into table 6 above gave the table 7 below:

Table 7: Output from fifth computation

\begin{tabular}{|c|c|c|c|c|c|}
\hline Weeks & $\begin{array}{c}\text { Maize } \\
\text { Flour }\end{array}$ & $\begin{array}{c}\text { Maize Bran } \\
\text { Flour }\end{array}$ & $\begin{array}{c}\text { Beans } \\
\text { Flour }\end{array}$ & $\begin{array}{c}\text { Cassava } \\
\text { Flour }\end{array}$ & $B_{. j}$ \\
\hline Week 1 & 8.09 & 8.00 & 7.73 & 7.71 & 31.53 \\
\hline Week 2 & 9.60 & 8.15 & 7.87 & 7.24 & 32.86 \\
\hline Week 3 & 8.14 & 7.75 & 7.60 & 7.64 & 31.13 \\
\hline Week 4 & 7.76 & 7.42 & 7.80 & 8.24 & 31.22 \\
\hline Week 5 & 7.17 & 7.68 & 7.21 & 8.03 & 30.09 \\
\hline Week 6 & 7.46 & 8.20 & 7.67 & $\hat{X}_{64}$ & 31.02 \\
\hline $\mathrm{T}_{\mathrm{i} .}$ & $\mathbf{4 8 . 2 4}$ & $\mathbf{4 7 . 2 0}$ & $\mathbf{4 5 . 8 8}$ & $\mathbf{3 8 . 8 6}$ & $\mathbf{1 8 0 . 1 6}$ \\
\hline
\end{tabular}

$$
\begin{gathered}
\hat{X}_{64}=\frac{6(23.33)+4(38.86)-180.16}{(6-1)(4-1)} \\
=\frac{115.26}{15}
\end{gathered}
$$

$\hat{X}_{64}=7.684 \cong 7.68$

Inserting $\hat{X}_{64}=7.68$ into table 7 above gave table 8 below:

Table 8: Output from sixth computation

\begin{tabular}{|c|c|c|c|c|c|}
\hline Weeks & $\begin{array}{c}\text { Maize } \\
\text { Flour }\end{array}$ & $\begin{array}{c}\text { Maize Bran } \\
\text { Flour }\end{array}$ & $\begin{array}{c}\text { Beans } \\
\text { Flour }\end{array}$ & $\begin{array}{c}\text { Cassava } \\
\text { Flour }\end{array}$ & $B_{. j}$ \\
\hline Week 1 & $\hat{\hat{X}}_{11}$ & 8.00 & 7.73 & 7.71 & 23.44 \\
& & & & \\
\hline Week 2 & 9.60 & 8.15 & 7.87 & 7.24 & 32.86 \\
\hline Week 3 & 8.14 & 7.75 & 7.60 & 7.64 & 31.13 \\
\hline Week 4 & 7.76 & 7.42 & 7.80 & 8.24 & 31.22 \\
\hline Week 5 & 7.17 & 7.68 & 7.21 & 8.03 & 30.09 \\
\hline Week 6 & 7.46 & 8.20 & 7.67 & 46.54 & 31.01 \\
\hline $\mathrm{T}_{\mathrm{i} .}$ & $\mathbf{4 0 . 1 3}$ & $\mathbf{4 7 . 2 0}$ & $\mathbf{4 5 . 8 8}$ & $\mathbf{4 6 . 5 7}$ & $\mathbf{1 7 9 . 7 5}$ \\
\hline
\end{tabular}

$$
\begin{gathered}
\hat{X}_{11}=\frac{6(23.44)+4(40.13)-179.75}{(6-1)(4-1)} \\
=\frac{121.41}{15}
\end{gathered}
$$

$$
\hat{X}_{11}=8.094 \cong 8.09
$$

Inserting $\hat{X}_{11}=8.09$ into table 8 above gave table 9 below:

Table 9: Output from seventh computation

\begin{tabular}{|c|c|c|c|c|c|}
\hline Weeks & $\begin{array}{c}\text { Maize } \\
\text { Flour }\end{array}$ & $\begin{array}{c}\text { Maize Bran } \\
\text { Flour }\end{array}$ & $\begin{array}{c}\text { Beans } \\
\text { Flour }\end{array}$ & $\begin{array}{c}\text { Cassava } \\
\text { Flour }\end{array}$ & $\mathrm{B}_{. j}$ \\
\hline Week 1 & 8.09 & 8.00 & 7.73 & 7.71 & 31.53 \\
\hline Week 2 & 9.60 & 8.15 & 7.87 & 7.24 & 32.86 \\
\hline Week 3 & 8.14 & 7.75 & $\hat{\wedge}$ & 7.64 & 23.53 \\
& & & $\hat{X}_{33}$ & & \\
& & & 7.80 & 8.24 & 31.22 \\
\hline Week 4 & 7.76 & 7.42 & 7.21 & 8.03 & 30.09 \\
\hline Week 5 & 7.17 & 7.68 & 7.67 & 7.68 & 31.01 \\
\hline Week 6 & 7.46 & 8.20 & 7.67 & \\
\hline $\mathrm{T}_{\mathrm{i} .}$ & 48.24 & 47.20 & 38.28 & 46.54 & 180.24 \\
\hline
\end{tabular}

$$
\begin{gathered}
\hat{\hat{X}}_{33}=\frac{6(23.53)+4(38.28)-180.24}{(6-1)(4-1)} \\
=\frac{114.06}{15}
\end{gathered}
$$

$$
\hat{X}_{33}=7.60
$$

Inserting $\hat{X}_{33}=7.60$ into table 9 above gave table 10 below:

\section{Volume 5 Issue 9, September 2016 www.ijsr.net}




\section{International Journal of Science and Research (IJSR) \\ ISSN (Online): 2319-7064 \\ Index Copernicus Value (2013): 6.14 | Impact Factor (2015): 6.391}

Table 10: Output from eighth computation

\begin{tabular}{|c|c|c|c|c|c|}
\hline Weeks & $\begin{array}{c}\text { Maize } \\
\text { Flour }\end{array}$ & $\begin{array}{c}\text { Maize Bran } \\
\text { Flour }\end{array}$ & $\begin{array}{c}\text { Beans } \\
\text { Flour }\end{array}$ & $\begin{array}{c}\text { Cassava } \\
\text { Flour }\end{array}$ & $\mathrm{B}_{. \mathrm{j}}$ \\
\hline Week 1 & 8.09 & 8.00 & 7.73 & 7.71 & 31.53 \\
\hline Week 2 & 9.60 & 8.15 & 7.87 & 7.24 & 32.86 \\
\hline Week 3 & 8.14 & 7.75 & 7.60 & 7.64 & 31.13 \\
\hline Week 4 & 7.76 & 7.42 & 7.80 & 8.24 & 31.22 \\
\hline Week 5 & 7.17 & 7.68 & 7.21 & 8.03 & 30.09 \\
\hline Week 6 & 7.46 & 8.20 & 7.67 & $\hat{\wedge}$ & 23.33 \\
& & & & $\hat{X}_{64}$ & \\
\hline $\mathrm{T}_{\mathrm{i} .}$ & 48.24 & 47.20 & 45.88 & 38.86 & 180.16 \\
\hline
\end{tabular}

$$
\begin{gathered}
\hat{\hat{X}}_{64}=\frac{6(23.33)+4(38.86)-180.16}{(6-1)(4-1)} \\
=\frac{115.26}{15}
\end{gathered}
$$

$$
\hat{X}_{64}=7.684 \cong 7.68
$$

The iteration stopped here and the estimated values are inserted into table 10 above thus, we obtained values for the original data: $\mathrm{X}_{11}=9.2, \mathrm{X}_{33}=7.78, \mathrm{X}_{64}=8.23$ :

$$
\hat{\hat{X}}_{11}=8.09 \hat{\hat{X}}_{33}=7.60 \hat{\hat{X}}_{64}=7.68
$$

Table 10: Results from all iterations

\begin{tabular}{|c|c|c|c|c|c|}
\hline Weeks & $\begin{array}{c}\text { Maize } \\
\text { Flour }\end{array}$ & $\begin{array}{c}\text { Maize } \\
\text { Bran Flour }\end{array}$ & $\begin{array}{c}\text { Beans } \\
\text { Flour }\end{array}$ & $\begin{array}{c}\text { Cassava } \\
\text { Flour }\end{array}$ & $B_{. j}$ \\
\hline Week 1 & $\mathbf{8 . 0 9}$ & 8.00 & 7.73 & 7.71 & 31.53 \\
\hline Week 2 & 9.60 & 8.15 & 7.87 & 7.24 & 32.86 \\
\hline Week 3 & 8.14 & 7.75 & $\mathbf{7 . 6 0}$ & 7.64 & 31.13 \\
\hline Week 4 & 7.76 & 7.42 & 7.80 & 8.24 & 31.22 \\
\hline Week 5 & 7.17 & 7.68 & 7.21 & 8.03 & 30.09 \\
\hline Week 6 & 7.46 & 8.20 & 7.67 & $\mathbf{7 . 6 8}$ & 31.01 \\
\hline $\mathrm{T}_{\mathrm{i} .}$ & $\mathbf{4 8 . 2 2}$ & $\mathbf{4 7 . 2 0}$ & $\mathbf{4 5 . 8 8}$ & $\mathbf{4 6 . 5 4}$ & $\mathbf{1 8 7 . 8 4}$ \\
\hline
\end{tabular}

\section{Conclusion}

It is helpful to note here that estimating missing values in randomized complete block design makes it possible to work with missing data probably due to death, misplacement and so on but it does not solve the problem in the actual sense because of the slightly different estimated values compared to the original data as it were. Consequently, statisticians try to ensure that the incidence of missing observation is minimized. Also in applied statistics, substitution of estimates for the missing data does not in any way recover the information (value) that is lost. As some experimenters may suggest, it merely attempt to reproduce the results obtained by an application of the least square method to the data that are present. The only perfect solution of the missing data problem is not to have them at all.

\section{Recommendations}

Having carried out the computation for the missing data manually, the following recommendations are made:
1) Applied statisticians should carry out a similar task on other experimental techniques to compare the results.

2) Researchers and other users of statistical data should employ updated and advanced statistical software packages, such as SAS, R-software for iterative computations of missing data to enable them obtain timely, cost effective and more reliable results.

\section{Acknowledgement}

I, Mr Ekholuenetale on behalf of my co-author, wholeheartedly appreciate Professor F.E. Okonofua of University of Medical Sciences, Ondo State, Nigeria for his mentoring role in building my research capability. I am deeply grateful Sir.

\section{References}

[1] Cochran WG, Cox M. Experimental Design. John Wiley and Sons Ltd, New York. 1957.

[2] Fisher RA. The Design of Experiments. $8^{\text {th }}$ Edition. Hafner Publishing Company, New York. 1966

[3] Hicks CR, Turner KV. Fundamental Concepts in the Design of Experiment. Oxford University Press, UK. 1973.

[4] Kempthorne O. The Design and Analysis of Experiments. Wiley, New York. 1952.

[5] Montgomery and Douglas. The Design and Analysis of Experiments. Wiley, New York. 1976.

[6] Montgomery, Douglas C. "Design and analysis of experiments" (8th Ed.). John Wiley \& Sons, 2012.

[7] Srivastava JN. Nonadditivity in row - column designs. J. Comb. Inform. System Sci; 1993. 18, 85-96

[8] Srivastava JN. A critique of some aspects of experimental design. In: Handbook of statistics, 13. S. Ghosh and C. R. rao (eds). Amsterdam: Elsvier Science. 1996: $309-341$

[9] Srivastava, J. N. and J. C. Wang. Row - Column designs: How and why nonadditivity may make them unfit for use. J. Statist. Plann. Inf; 1998. 73, 277 - 315

[10] Yates F. Design and Analysis of Factorial experiment. Tech. Comm. No. 35, Imperial Bereau of Soil Science, London. 1937. 